\title{
SCIENTIFIC REPORTS

\section{OPEN Optimisation of robust singleplex and multiplex droplet digital PCR assays for high confidence mutation detection in circulating tumour DNA}

\author{
Vicky Rowlands $\mathbb{B D}^{1}$, Andrzej J. Rutkowski ${ }^{1,3}$, Elena Meuser ${ }^{1}$, T. Hedley Carr $\mathbb{D}^{1}{ }^{1}$, \\ Elizabeth A. Harrington ${ }^{1} \&$ J. Carl Barrett ${ }^{2}$
}

Liquid biopsies offer the potential to monitor cancer response and resistance to therapeutics in near real-time. However, the plasma cell free DNA ( $C f D N A$ ) level can be low and the fraction of circulating tumour DNA (ctDNA) bearing a mutation - lower still. Detection of tumour-derived mutations in ctDNA is thus challenging and requires highly sensitive and specific assays. Droplet digital PCR (ddPCR) is a technique that enables exquisitely sensitive detection and quantification of DNA/RNA markers from very limiting clinical samples, including plasma. The Bio-Rad QX200 ddPCR system provides absolute quantitation of target DNA molecules using fluorescent dual-labelled probes. Critical to accurate sample analysis are validated assays that are highly specific, reproducible, and with known performance characteristics, especially with respect to false positives. We present a systematic approach to the development and optimisation of singleplex and multiplex ddPCR assays for the detection of point mutations with a focus on ensuring extremely low false positives whilst retaining high sensitivity. We also present a refined method to determine cfDNA extraction efficiency allowing for more accurate extrapolation of mutational levels in source samples. We have applied these approaches to successfully analyse many ctDNA samples from multiple clinical studies and generated exploratory data of high quality.

Monitoring of response and resistance to therapies in cancer patients at the molecular level presents many challenges. Traditional tumour biopsies are invasive and only a portion of a tumour is assessed at a specific time point representing a snapshot of a patient's disease. Body cells shed fragments of DNA into the circulatory system (cell-free DNA/cfDNA, sometimes called circulating cell-free DNA/ccfDNA). In cancer patients, a fraction of cfDNA will be of tumour origin (designated circulating tumour DNA/ctDNA) $)^{1,2}$. ctDNA is characterised by cancer-specific mutations and it can be monitored by sampling blood (usually the plasma component), representing a "liquid biopsy". There is a huge and ever-growing body of publications documenting the usefulness of ctDNA as a biomarker in longitudinal tracking of mutation status and disease burden throughout treatment $\left(\right.$ reviewed $\mathrm{in}^{2-6}$ ). Non-invasive liquid biopsies offer the potential to sample frequently to monitor genomic biomarkers for patient response and identify emergence of mutations conferring resistance to cancer therapeutics. This has been documented for many genes, e.g. the T790M mutation in EGFR (driving resistance to gefitinib and erlotinib $^{7}$ ) or multiple KIT mutations (imatinib ${ }^{8}$ ), reviewed in $^{2,4,9}$.

Detection of tumour-derived mutations in ctDNA is challenging because the tumour DNA is often at a very low concentration, short, fragmented, and diluted by the presence of a background of non-mutant DNA (both tumour and non-tumour origin). Analysis thus requires highly sensitive and specific assays. Various DNA sequencing techniques can be utilized to identify and monitor mutations in ctDNA, each with their own

${ }^{1}$ Translational Medicine, Oncology R\&D, AstraZeneca, Cambridge, UK. ${ }^{2}$ Translational Medicine, Oncology R\&D, AstraZeneca, Boston, USA. ${ }^{3}$ Present address: Medicines Discovery Catapult, Block 35, Mereside, Alderley Park, Alderley Edge, Cheshire, SK10 4TG, UK. Vicky Rowlands and Andrzej J. Rutkowski contributed equally. Correspondence and requests for materials should be addressed to V.R. (email: vicky.rowlands@astrazeneca.com) 
advantages and disadvantages ${ }^{2,10-13}$. In the non-sequencing space, digital PCR, most notably droplet digital PCR (ddPCR), is a technique which allows for highly sensitive and specific detection of mutations. For digital PCR the assays are limited to specific single mutations or sets of highly related mutations at the same locus ${ }^{14}$. By the nature of PCR, its two primers (and optionally a probe) target a very specific sequence. A few sets of primers and probes targeting several genomic regions can be mixed together in one PCR reaction to produce multiplex PCR. This multiplexing, however, comes with multiple challenges such as varying efficiency of individual assays, different primer annealing temperatures, possible oligonucleotide cross-dimerisation and accurate separation of fluorescent signals from a limited number of reporter dyes with overlapping emission spectra ${ }^{15}$. For these reasons, the analysis of broader genomic regions using ddPCR is not possible. However, it is possible to develop discriminatory multiplex ddPCR assays that enable very rapid and cost-effective monitoring for a limited number of mutations in serial plasma samples. Such assays can be very informative for serial analysis after target mutations are identified via broader sequence profiling of tumour tissue or a baseline ctDNA sample. Droplet digital PCR allows calculation of absolute DNA quantity based upon the number of positive and negative droplets observed. This is done according to the Poisson distribution without the need for external reference standards or controls ${ }^{14}$. However, the performance of every ddPCR assay is different. For a given assay, the relative fluorescence signal that defines a true positive droplet from a negative droplet, and the extent to which droplets tend to fall between those values, can vary enormously dependent on multiple factors, including target locus sequence context, performance of the amplicon, cycling conditions employed, and concentrations of key reagents. In addition, the relative frequency of false positive droplets (usually attributable to polymerase error early during the thermal cycling phase) can vary significantly depending on the base change and sequence context. With assays intended to be used for detection of mutations down to single digit copies per reaction, it is critical that the performance of every assay is thoroughly understood. With assays intended to test for multiple mutations simultaneously these challenges are multiplied, and performance of each assay will be different between singleplex and multiplex even if using the same reagents at the same concentrations and under the same reaction conditions.

Here we describe how we have developed and systematically validated robust exploratory singleplex and multiplex ddPCR assays for the detection and monitoring of tumour-derived point mutations in human plasma using the Bio-Rad QX200 platform with probe-based detection. To minimise the volume of clinical sample used for screening for mutations, singleplex assays can be combined together in a multiplex assay that enables rapid and cost-effective analysis of serial plasma time points for a range of mutations. Our approach is based on several years of experience of utilising this platform for these purposes. In addition, we also present a refinement of a previously reported method for calculating an accurate quantification of mutant copies in the original plasma sample utilising an externally spiked control DNA to correct for variation in cfDNA extraction efficiencies ${ }^{16,17}$.

\section{Materials and Methods}

Samples sourced and informed consent. Plasma samples were obtained from a commercial vendor and early phase clinical studies.

SeraLab (now a division of BioreclamationIVT) are an AstraZeneca approved supplier, in that AstraZeneca have assurance that any plasma sample supplied has been collected ethically, with consent for research, and in accordance with all regulatory requirements. AstraZeneca holds a UK Human Tissue Authority Licence (Licence Number 12109) and Research Tissue Bank Ethics Approval for research involving human tissue (RTB Ethics reference 17/NW/0207).

All patients provided written informed consent before any study-specific procedures, sampling, and analyses from an early phase clinical study as detailed in the clinical study protocol (NCT03101839).

Cell-free DNA isolation from plasma. Plasma samples processed from Streck Cell-Free DNA BCT or $\mathrm{K}_{2}$ EDTA blood collection tubes were thawed on wet ice. cfDNA was isolated from up to $2 \mathrm{ml}$ plasma using a Maxwell ${ }^{\circledR}$ RSC instrument with ccfDNA Plasma Kit (AS1480) from Promega (Madison, Wisconsin, USA), KingFisher Flex System from Thermo Fisher Scientific (Waltham, MA, USA) with MagBind ${ }^{\circledR}$ cfDNA Kit (M329801) from Omega BioTek (Georgia, USA) or QIAamp Circulating Nucleic Acid Kit (1017647) with the QIAvac 24 Plus vacuum manifold from Qiagen (Manchester, UK) according to the manufacturers' protocols. In most cases, plasma samples were spiked with 20,000 copies of a short, synthetic double stranded DNA fragment (gBlock ${ }^{\circledR}$ Gene Fragments) (Integrated DNA Technologies Inc (IDT), Leuven, Belgium), prior to extraction. gBlock ${ }^{\circledR}$ Gene Fragments are sequence verified double stranded DNA fragments, which can be utilized in a variety of molecular biology applications. The XenT gBlock contains a sequence from Xenopus tropicalis that does not share homology with any human DNA sequence and is assumed to perform similarly to real cfDNA in extraction protocols. To quantify the recovered copies of XenT post extraction, a dual XenT/RPP30 ddPCR assay was developed. The XenT ddPCR assay was designed by IDT and the RPP30 assay was purchased from Bio-Rad (\#dHsaCP2500350). RPP30 is an essential gene that encodes the human Ribonuclease P Protein Subunit P30 - this gene is highly conserved, unique in the genome, rarely (if ever) impacted by copy number changes and is thus a common choice of control gene for copy number studies ${ }^{14}$. The RPP30 assay was used to control for total cfDNA (of tumour and non-tumour origin). To estimate the cfDNA extraction efficiency, XenT was quantified in both the XenT gBlock solution used for spike-in and in the extracted samples. Eluates were stored in DNA low-bind tubes (DNA LoBind ${ }^{\circledR}$, Eppendorf or SC Micro Tube DNA LB, Sarstedt) at $-20^{\circ} \mathrm{C}$.

Droplet digital PCR workflow. ddPCR was performed using the QX200 AutoDG Droplet Digital PCR System (Bio-Rad, California, USA) as per the manufacturer's protocol with minor modification, as described below and in Results. All preparation and reaction set up was performed in a dedicated pre-PCR room and PCR hood. 


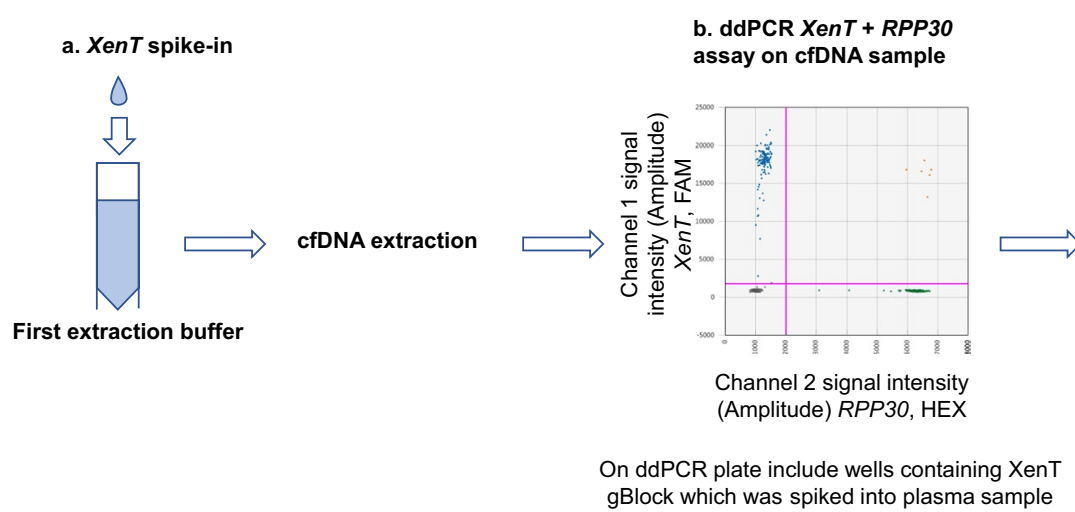

c. Calculation of cfDNA extraction efficiency

Using data output in ddPCR output column for 'Copies/20 L L well' calculate:

a. Number of XenT copies in eluted cfDNA

b. Number of XenT copies spiked in to plasma

$\%$ Extraction efficiency $=a / b * 100$

Extraction efficiency factored in when calculating mutant copies per mL plasma

Figure 1. Refinement of a method to assess cfDNA extraction efficiency from plasma. (a) A defined number of XenT gBlock copies were spiked into each plasma sample prior to extraction, (b) ddPCR was performed using the developed XenT/RPP30 assay to (c) calculate cfDNA extraction efficiency.

Custom assays were designed by IDT incorporating locked nucleic acid (LNA) bases ${ }^{18}$ in varying numbers into each probe to increase discrimination and sensitivity ${ }^{18}$. LNA-bearing PrimeTime ${ }^{\circledR}$ probes containing either a $5^{\prime}-\mathrm{FAM}^{\mathrm{TM}}$ or $5^{\prime}-\mathrm{HEX}^{\mathrm{TM}}$ reporter dye and $3^{\prime}$ Iowa Black ${ }^{\circledR}$ Fluorescent quencher were HPLC purified. Primers were ordered from Eurogentec (Liège, Belgium) (SePOP desalted). All oligonucleotides were resuspended in TE buffer $\left(10 \mathrm{mM}\right.$ Tris- $\mathrm{HCl}(\mathrm{pH} 8.0), 0.1 \mathrm{mM}$ EDTA) and stored at $-20^{\circ} \mathrm{C}$. Probe and primer details for all assays can be found in Supplementary Table 1.

Each $22 \mu \mathrm{L}$ ddPCR reaction contained $11 \mu \mathrm{L}$ of $2 x$ ddPCR SuperMix for probes (no dUTP) (Bio-Rad), template DNA, forward and reverse primers, and FAM- and HEX-labelled probes at concentrations defined during assay optimisation. Reactions were prepared in a semi-skirted 96 well plate (Eppendorf). Following droplet generation on the AutoDG, the plate was sealed with a pierce-able foil heat seal (Bio-Rad) and PCR performed on a $\mathrm{T}_{100}{ }^{\mathrm{TM}}$ or C1000 Touch ${ }^{\mathrm{TM}}$ thermal cycler (Bio-Rad). After PCR, the plate was incubated at $12^{\circ} \mathrm{C}$ for a minimum of 4 hours. The plate was then incubated at room temperature for 10 minutes prior to being read using a QX200 droplet reader (Bio-Rad).

Every ddPCR run included negative template controls (NTCs) and positive template controls (PTCs) run in triplicate or quadruplicate. NTCs included water used for reaction mixes, TE buffer used for dilution of oligonucleotides, and elution buffer used in the cfDNA extraction (for runs that included cfDNA samples). PTCs included commercially acquired wild-type DNA from male human cells (Promega), gDNA Reference Standards (Horizon Discovery, Cambridge, UK) containing the respective mutations, or gBlocks. We used gBlocks of length $150-170 \mathrm{bp}$, which represents the approximate size of the major cfDNA peak in human plasma ( 167 bp), though reports vary ${ }^{19,20}$. gBlocks were routinely diluted to about 7000 copies per microlitre (designated 'dilution C') from which subsequent 10-fold dilutions (dilutions D and E) were prepared. PTCs were quantified at the assay development stage and thus served as subsequent controls for consistency of assay performance.

Thresholds were manually set for each sample using acceptance criteria defined during the optimisation of each assay. QuantaSoft ${ }^{\mathrm{TM}}$ Analysis Pro software (version 1.0.596) or QuantaSoft ${ }^{\mathrm{TM}}$ software (version 1.7.4) was used to assign positive/negative droplets and convert counts to a copies $/ \mathrm{ml}$ or fractional abundance value (\% allelic frequency). In most cases, conversion included correction for extraction efficiency based on results of analysis of the XenT gBlock synthetic spike-in.

\section{Results}

Refinement of a method to assess cfDNA extraction efficiency from plasma. Various methods are in use to purify cfDNA from plasma. These are typically either column or magnetic-bead based approaches. Although purification of cfDNA is a critical step prior to any genomic assay, increasing number of publications report that extraction efficiencies can vary greatly, depending on several factors, from the kind of sample collection tubes to extraction kits ${ }^{21-25}$. Variation between methods and performance differences from day to day or batch to batch might skew the final estimate of cfDNA in the original sample. We therefore refined a method to assess extraction efficiency using a gBlock spiked into plasma prior to cfDNA isolation (Fig. 1a) ${ }^{16}$. A dual $X e n T / R P P 30$ ddPCR assay was used (Fig. 1b), as described in Materials and Methods. To estimate the cfDNA extraction efficiency, XenT was quantified in both the XenT gBlock solution used for spike-in and in the extracted samples. The former was used to calculate the theoretical number of XenT copies representing a $100 \%$ efficient extraction and the latter was a measure of the actual extraction efficiency. The derived extraction efficiency was factored in when calculating DNA copies/ml plasma (Fig. 1c) in ddPCR assays testing for human WT and mutant DNA. The development of this method also allows for direct DNA extraction efficiency comparison across different extraction platforms. For example, in a series of 228 extractions, we determined that the cfDNA extraction efficiency using the KingFisher Flex instrument was $64.3 \pm 12.2 \%$ (range 34.5-92.8\%). We found that the level of detected WT DNA was not correlated with the extraction efficiency. We compared the top and bottom quartile of samples with regards to the total amount of extracted cfDNA (as determined by the number of detected RPP30 copies; Supplementary Fig. 1). We found that the extraction efficiencies were $63.3 \pm 13.0 \%$ (range 45.7-88.1\%) and $64.1 \pm 12.2 \%$ (range 34.6-92.0\%) for the top and bottom quartiles, respectively (unpaired two-tailed t-test 
a. 1D plot

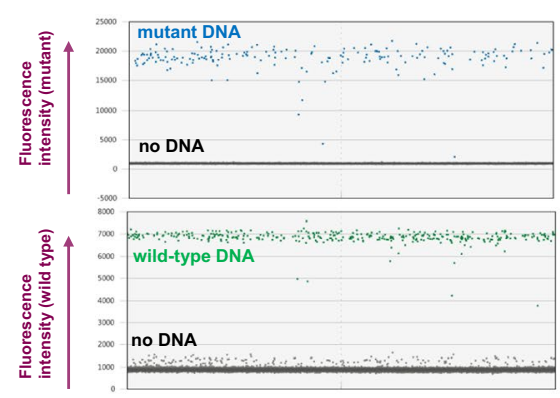

b. 2D plot

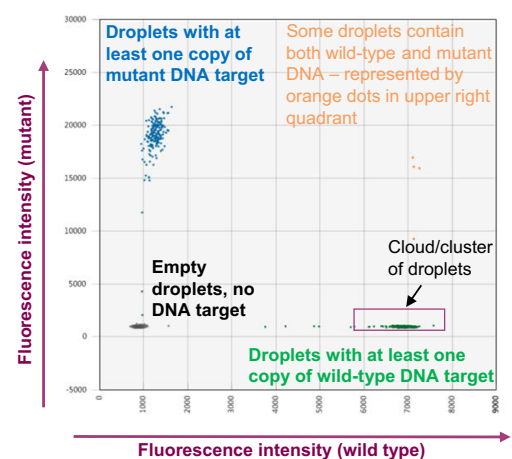

c. 'Rain'

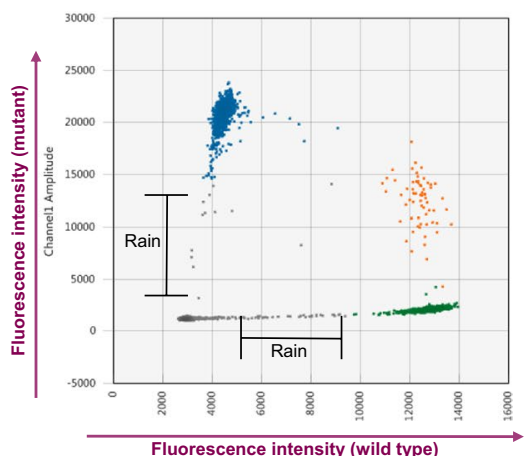

Figure 2. Key features and concepts of ddPCR data. Data can be visualised in a (a) 1D or (b) 2D plot. Approximately 20k droplets are analysed. Fluorescence of each droplet is measured and for analysis projected on a $1 \mathrm{D}$ or $2 \mathrm{D}$ plot. Each dot on the figure represents one droplet containing at least one copy of DNA target: mutant (blue), wild type (green) or no DNA (black). (b) Some droplets can contain both wild type and mutant DNA targets, they are represented by orange dots in the top right quadrant of the 2D plot. (c) 'Rain' defines those droplets which occur between the clusters of negative and positive droplets, generally due to a poorly optimised assay.

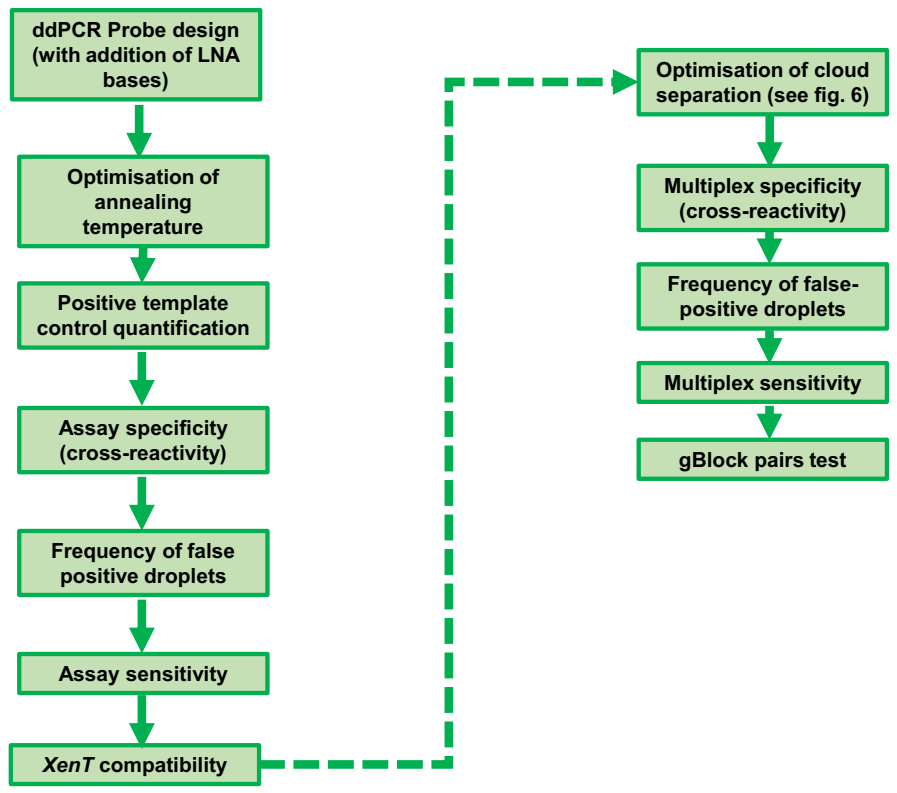

Figure 3. ddPCR assay optimisation workflow. The workflow highlights the key stages in optimisation of a sensitive, specific and reproducible ddPCR assay for (a) singleplex and (b) multiplex assays. Steps in (a) must be completed before (b) multiplex assay optimisation workflow commences. For an explanation of the purpose of each stage, refer to Supplementary Tables 2 and 3.

$\mathrm{p}=0.1127$ ). Whilst there seems to be no difference in extraction efficiencies between samples with high vs low detectable levels of RPP30, this still highlights the necessity for approximating the extraction efficiency to get an estimate of true cfDNA and ctDNA levels in plasmas containing very high or very low cfDNA levels.

ddPCR assay optimisation workflow. A description of key ddPCR data analysis concepts are presented in Fig. 2. Our approach to optimising highly sensitive, specific and reproducible ddPCR assays are outlined in Fig. 3, described below, and in Supplementary Tables 2 and 3.

ddPCR probe design. The incorporation of melting temperature (Tm)-enhancers (such as LNA bases or similar modifications) into each probe was highly beneficial. The design work was generally undertaken by IDT, but users can design LNA probes following the QX200 Applications Guide and in You et al. ${ }^{18}$. LNA inclusion was an important factor for optimal performing assays. By comparison to Bio-Rad's assays, we found that using 

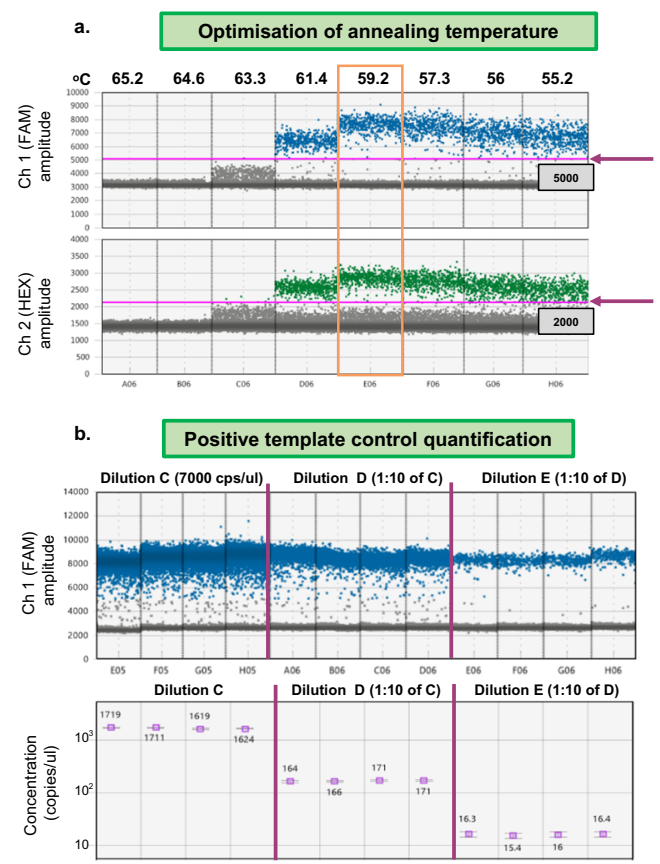

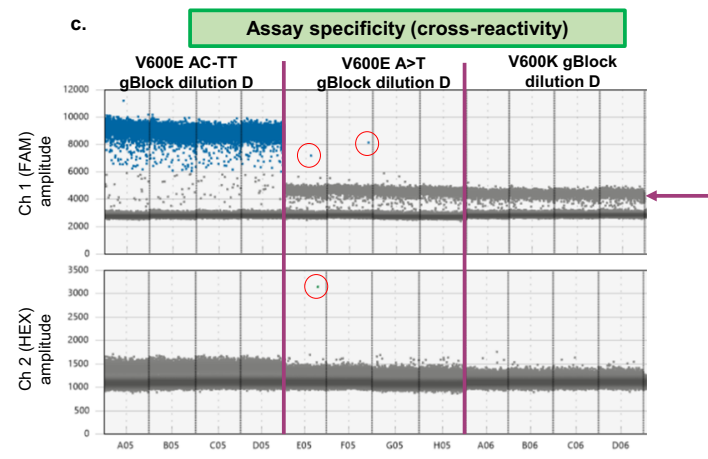

Figure 4. Results from optimisation of BRAF V600E (AC > TT) ddPCR assay for annealing temperature, positive template control quantification and assay specificity (cross reactivity) optimisation steps. (a) Using the V600E AC $>$ TT gBlock positive template control in an annealing temperature gradient, optimum separation of negative and positive droplets for both channels was achieved at $59.2^{\circ} \mathrm{C}$ (yellow box) and the fluorescence cutoffs indicated (arrows and grey boxes). (b) The V600E AC $>$ TT gBlock 10-fold dilution series (diluted to 7000 copies/ul, 700 copies/ul and 70 copies/ul in Dilution C, D and E, respectively) produced the expected results upon quantification using the previously determined thresholds and (c) there was no significant cross reactivity to any other BRAF V600 mutations in the panel. The arrow highlights low-level cross reactivity to the V600E A > T and V600K gBlocks, below the threshold for positivity. Red circles highlight false positive droplets which were present at acceptably low levels. Cps - copies.

LNA-containing probes allowed for shorter probe length with higher Tm's, in addition to providing the user with the exact sequence of all primers. The latter is an important advantage when the user aims to multiplex assays and sequence details are needed to assess oligonucleotide cross-annealing. Additionally, when Bio-Rad does not offer an assay for a sequence/variant of interest, using assays that were all designed by the end user provides for consistency of their chemistries. This helps to achieve tighter clusters in the resulting $2 \mathrm{D}$ plots, better separation between mutant and wild-type clusters, reduced "rain" (droplets which occur between the clusters of negative and positive droplets), and allows for precise thresholds to be set for calling an assay positive for a target mutation ${ }^{26}$. For illustration of the concepts, see Fig. 2.

Optimisation of the annealing temperature using a thermal gradient. Optimisation of annealing temperature was critical for reaction specificity. We found that primers and probes at final concentrations of $1.8 \mu \mathrm{M}$ and $500 \mathrm{nM}$, respectively, were optimal, with an annealing temperature gradient of $55^{\circ} \mathrm{C}-65^{\circ} \mathrm{C}$ (Fig. 4a). For the selected annealing temperature, thresholds were selected that separated positive and negative droplets in both channels. As a general guideline, these were about a quarter of the way between the median fluorescence levels of negative and positive droplets. We selected the optimal annealing temperature based on the highest temperature that produced the best separation of negative and positive droplets, taking both channels into consideration. When assays were being developed for more than one mutation, selection of a single annealing temperature that was optimal for all assays was advantageous for subsequent multiplex development, though this was not always possible.

Singleplex assay optimisation. Singleplex assays were optimised using a stepwise approach described in Fig. 3 and Supplementary Table 2. Figures 4 and 5 show the results of optimisation of a BRAF V600E (AC $>$ TT) assay. The quantification of the gBlock positive template control dilutions produced the expected result and there was no significant cross reactivity to other BRAF V600 assays in the panel (Fig. 4b,c). 84 wells of WT DNA were run to assess frequency of false positive droplets; 1 well had 1 false positive FAM droplet, 83 wells had 0 false positive FAM droplets, giving a false positive rate of 1 false positive in $865,553 \mathrm{WT}$ copies $(0.0000012 \%)$ (Fig. $5 \mathrm{a})$. In terms of assay sensitivity, the assay performed well and detected very low levels of V600E AC $>$ TT mutation even in the presence of a high number of WT copies (Fig. 5b). XenT gBlock was not recognised by the V600E AC $>$ TT assay, XenT/RPP30 assay did not bind to V600E AC > TT gBlock, and presence of the XenT gBlock did not significantly affect the detection of very low copies of mutant V600E AC $>$ TT (Fig. 5c). 
a. Frequency of false positive droplets

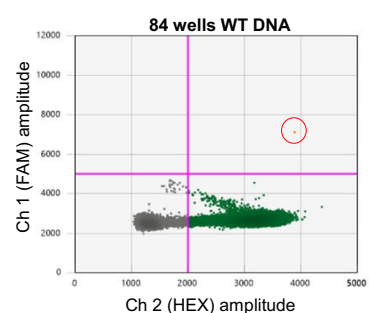

\begin{tabular}{|c|c|}
\hline \multicolumn{2}{|c|}{ False positive droplets in 84 wells } \\
\hline $\begin{array}{c}\text { \# wells with } 0 \text { false } \\
\text { positive droplets }\end{array}$ & 0 \\
\hline $\begin{array}{c}\text { \# wells with } 1 \text { false } \\
\text { positive droplet }\end{array}$ & 1 \\
\hline
\end{tabular}

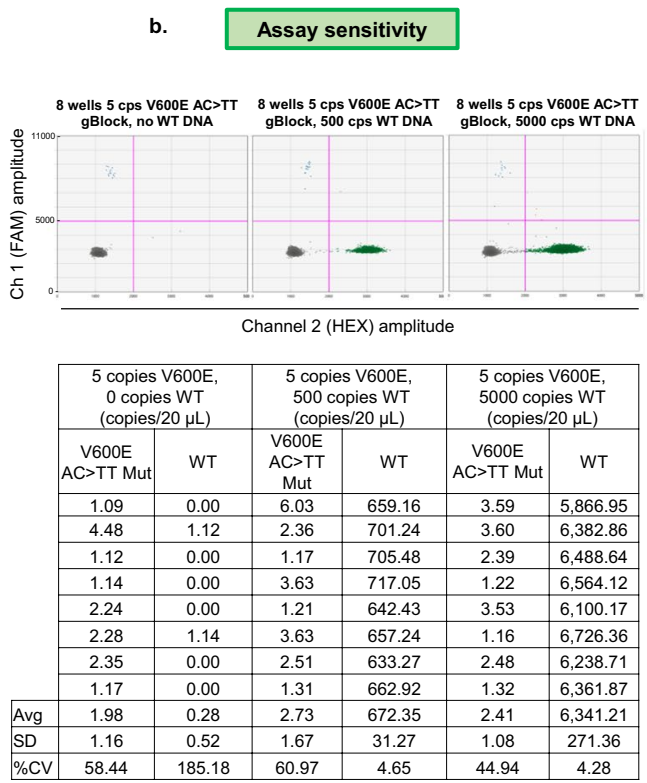

c.

XenT compatibility

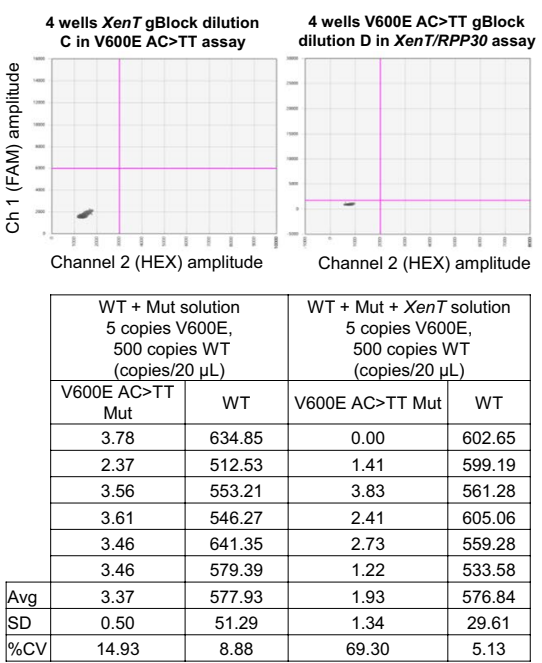

Figure 5. Results from optimisation of BRAF V600E (AC $>$ TT) ddPCR assay for frequency of false positive droplets, assay sensitivity and XenT compatibility optimisation steps. (a) 84 wells of WT DNA were run. At the previously determined thresholds, 1 well had 1 FAM false positive droplet, 83 wells had 0 FAM false positive droplets. The red circle highlights the false positive droplet. (b) At the previously determined thresholds, the BRAF V600E AC > TT ddPCR assay showed high sensitivity and detected very low levels of V600E AC $>$ TT mutation ( 5 copies) even in the presence of high numbers of WT copies (up to 5000 copies WT). Two spurious HEX positive droplets were observed in the absence of WT DNA potentially due to polymerase error. Full details reported as copies $/ 20 \mu \mathrm{l}$ as well as measurement summaries are presented in the table below the respective $2 \mathrm{D}$ plots. (c) The XenT assay is fully compatible with the V600E AC > TT assay. The XenT gBlock was not recognised by the V600E AC > TT assay, and vice versa, the XenT/RPP30 assay did not bind to the V600E AC > TT gBlock. The table below the 2D plots demonstrates that presence of the XenT gBlock did not significantly affect the detection of very low copies of mutant V600E AC $>$ TT. All measurements are reported as copies $/ 20 \mu \mathrm{l}$ using the V600E AC $>$ TT assay with either a mixture of 5 copies of V600E AC $>$ TT gBlock in a background of 500 copies WT DNA (WT + Mut) or this mixture additionally containing the XenT gBlock $(\mathrm{WT}+\mathrm{Mut}+\mathrm{XenT}) . \mathrm{Cps}-$ copies.

Multiplex assay optimisation with examples of assay development and use. Multiplex assays were optimised to ensure every mutation was represented on a $2 \mathrm{D}$ plot by an individual cloud that did not overlap with any other cloud. The performance of the assays in the presence of DNA with different combinations of the target mutations was assessed, as well as the baseline rate of false positive droplets from non-mutant (WT) genomic DNA. The process of developing the optimal probe blend and cycling conditions as well as the validation of multiplex specificity and sensitivity is illustrated in Fig. 3, Supplementary Table 3 and Fig. 6.

Multiplexing of four PIK3CA assays was achieved by modifying probe concentrations and by the combination of FAM and HEX fluorescent labels (Fig. 7). Probe concentrations in the final assays were as follows: $250 \mathrm{nM}$ exon $9 \mathrm{WT}, 250 \mathrm{nM}$ exon $20 \mathrm{WT}, 250 \mathrm{nM}$ E542K, $125 \mathrm{nM}$ E545K, 375 nM H1047L and $250 \mathrm{nM}$ H1047R. Combination of H1047L mutant FAM probe with H1047L mutant HEX probe shifted the cloud to the right on the 2D plot providing optimal separation from the other mutant clouds (Fig. 7a-c). Figure 7d shows how the optimised assay performs when wild-type DNA was also present.

To demonstrate the utility of the assays, we purchased 96 clinical plasma samples from a commercial vendor (BioIVT, UK). The samples were from oestrogen receptor positive breast cancer patients who previously responded to aromatase inhibitor treatment and then relapsed. Of the 96 samples, 5 were truly positive for at least one PIK3CA mutation (5.2\%), which was a lower incidence than expected (Fig. $8 \mathrm{a})^{27}$. When 'borderline' positive cases were included (those samples with one or two mutant copies), this raised the total to 20/96 (20.8\%) which is approximately the expected frequency within the population ${ }^{27}$. One ctDNA sample contained more than one PIK3CA mutation (Fig. 8b). This sample was also positive for ESR1 Y537S. These results demonstrate the utility of a multiplex ddPCR assay in assessing potential polyclonality/disease heterogeneity whilst limiting the required amount of precious clinical material.

For another project we required high quality assays that cover 14 prevalent $K R A S$ mutations across multiple disease indications whilst sparing ctDNA amounts and limiting lab time required. No appropriate assays existed to suit our requirements (see Discussion) and thus, following the guidelines described herein, three multiplex assays were devised and optimised (Fig. 9a-c). The assays detected and discriminated between four or five different KRAS mutations in each reaction. Each multiplex was extensively experimentally validated according to the principles described above to be highly sensitive, specific and reproducible. The worst performing assay gave a 


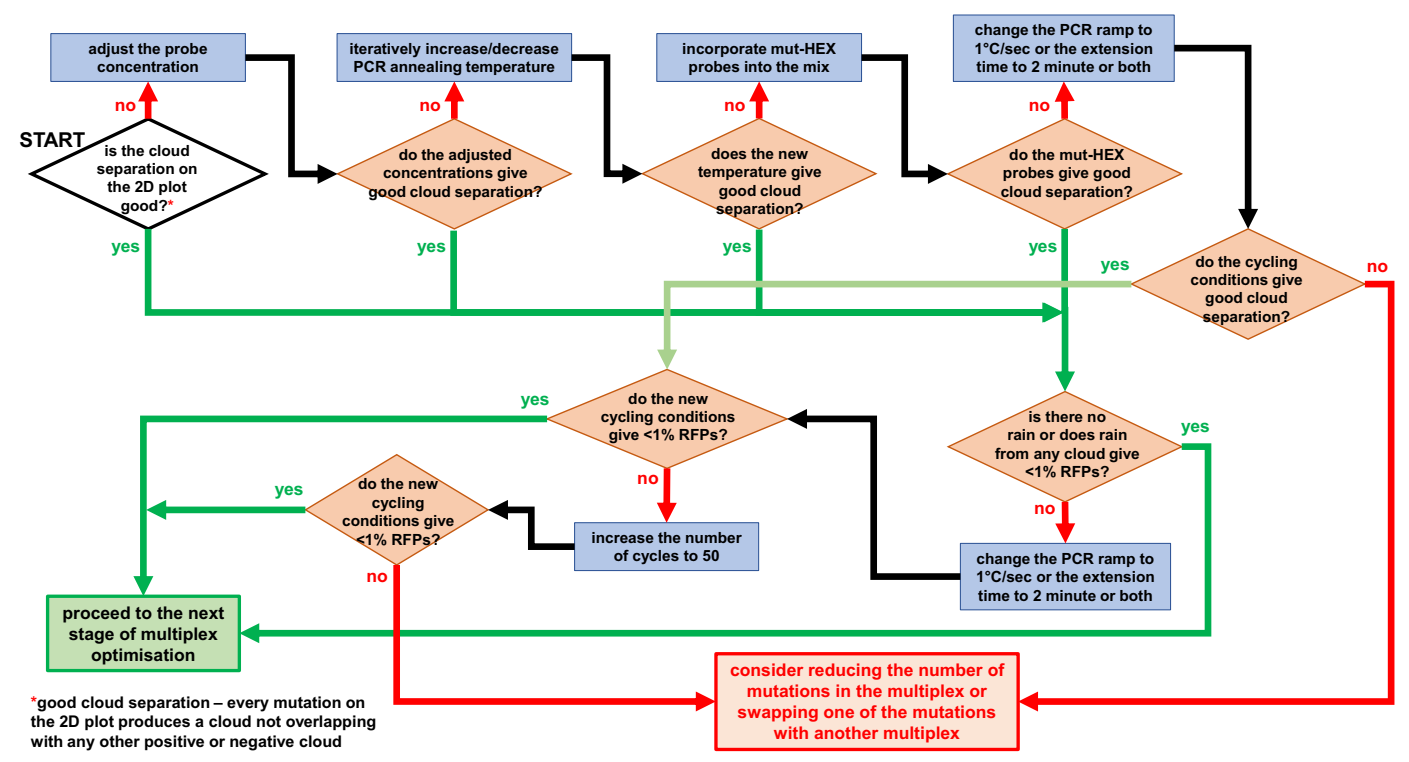

Figure 6. Optimisation of cloud separation in a multiplex ddPCR assay.

\section{a. FAM labelled probes only}

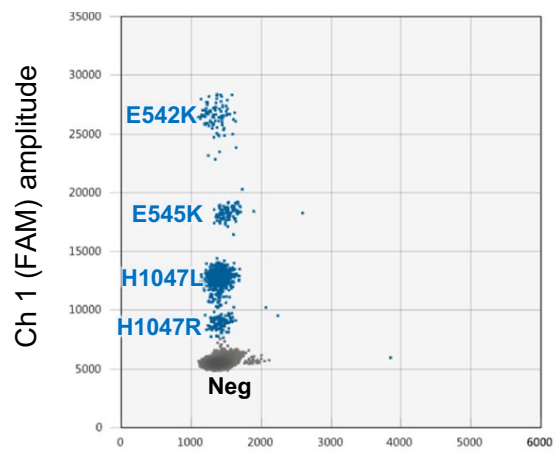

Ch 2 (HEX) amplitude

\section{c. H1047L FAM:HEX at 1:1 ratio}

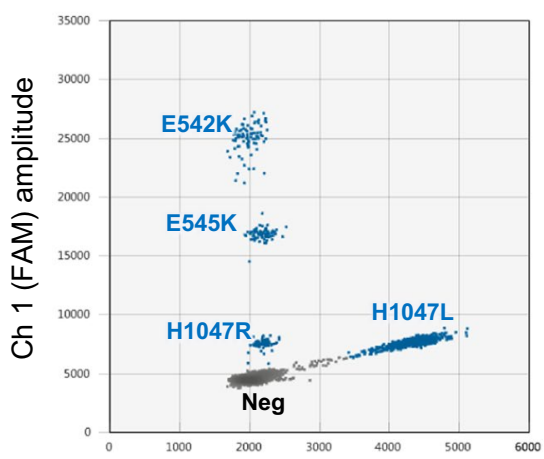

Ch 2 (HEX) amplitude

\section{b. H1047L FAM:HEX at 3:1 ratio}

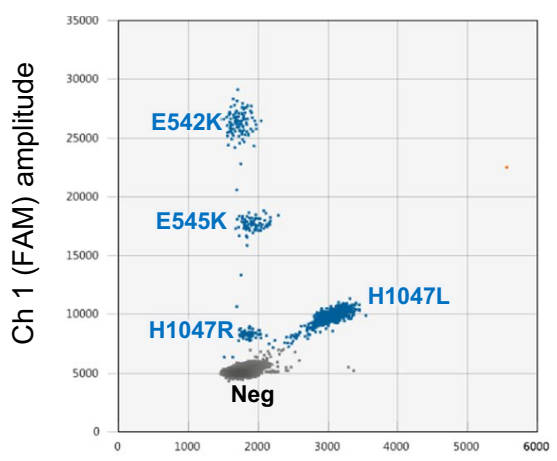

Ch 2 (HEX) amplitude

\section{d. Cloud distribution with wild-type DNA present}

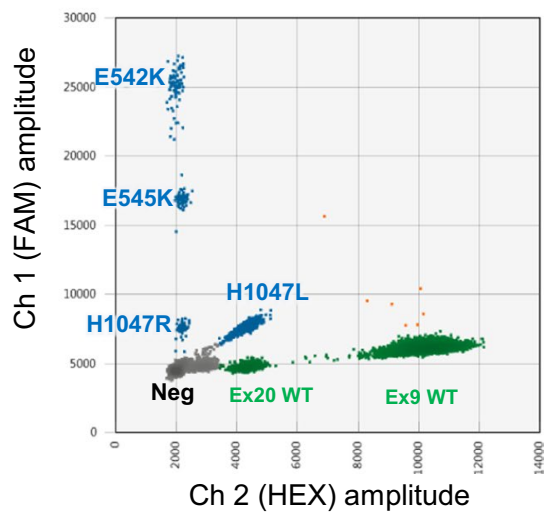

Figure 7. Combination of FAM and HEX labelled probes to optimise mutant cloud separation in a PIK3CA multiplex assay. (a) Initial cloud distribution of the multiplex reaction was not optimal. (b) Addition of two H1047L mutant probes labelled with HEX and FAM dyes in 1:3 ratio moved the associated cloud to the right and in (c) 1:1 ratio, further moved the cloud to the right and lowered the channel 1 amplitude. (d) The final optimised cloud distribution in the presence of non-mutant DNA is shown. Neg - double negative droplets. Ex20 WT - PIK3CA Exon 20 WT Positive droplets. Ex9 WT - PIK3CA Exon 9 WT Positive droplets. 
a. ddPCR results summary

\begin{tabular}{|c|c|c|}
\hline Sample & PIK3CA status by ddPCR & $\begin{array}{c}\text { ESR1 Y537C/N/S and D538G } \\
\text { status by ddPCR }\end{array}$ \\
\hline 2 & H1047R +ve & - ve \\
\hline 35 & E545K +ve,,$<3$ droplets for H1047R & Y537C +ve \\
\hline 57 & E542K +ve & $-v e$ \\
\hline 77 & $\mathrm{E} 545 \mathrm{~K}+\mathrm{ve},<3$ droplets for E542K & $-\mathrm{ve}$ \\
\hline 96 & $\begin{array}{c}\mathrm{E} 542 \mathrm{~K}+\mathrm{ve}, \mathrm{H} 1047 \mathrm{R}+\mathrm{ve}, \mathrm{H} 1047 \mathrm{~L}+\mathrm{ve}, \\
<3 \text { droplets for E545K }\end{array}$ & $\begin{array}{c}\text { Y537S +ve, <3 droplets for } \\
\text { D538G }\end{array}$ \\
\hline
\end{tabular}

b. Patient sample positive for more than one PIK3CA mutation (sample 96)

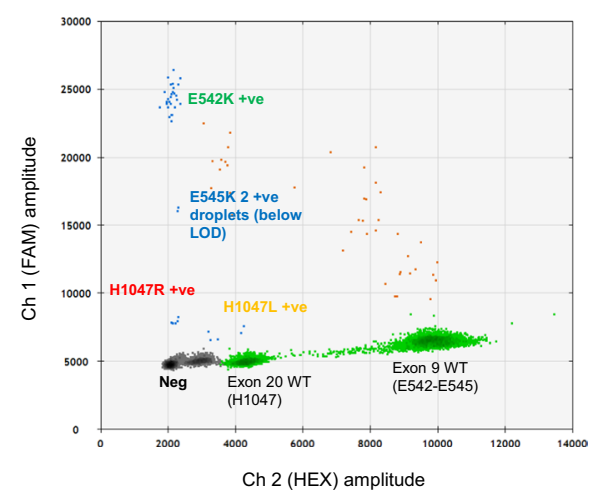

Figure 8. ddPCR results on a cohort of commercial clinical ER + ve breast cancer patients. (a) Results from screening $96 \mathrm{ctDNA}$ samples for PIK3CA and ESR1 mutation. Samples found to be positive for PIK3CA mutation were tested for the presence of an ESR1 mutation (Y537C/N/S and D538G). (b) Commercial clinical PIK3CA mutant positive sample positive for E542K, H1047R and H1047L mutations and borderline for E542K (sample 96).

false positive rate below $0.0027 \%$ (or one false positive mutant copy reported in 36,400 wild type genome copies). All other assays had significantly lower false positive rates.

When testing patient plasma samples, multiplex assays always included a positive control that was a mixture of DNA fragments (e.g. gBlocks) representing every mutation targeted by that assay to assure no abnormalities in cloud distribution. Multiplex analysis found several samples to be positive for KRAS G12D mutation and these samples were further tested by the G12D singleplex assay. Supplementary Table 4 shows a multiplex and singleplex ddPCR analysis comparison for seven samples. The data show that the copies $/ \mathrm{ml}$ calls in both types of analysis were similar for every sample, with a maximum coefficient of variation of $17.12 \%$ (range $2.83-17.12 \%$ ). These results further demonstrated the utility of a multiplexed ddPCR assay in assessing disease heterogeneity whilst limiting the amount of precious clinical material required.

Because of the nature of multiplex results, droplets were not counted as mutation-positive simply based on FAM thresholds (as for singleplex assays). Instead, during optimisation, zones on the 2D plot were associated with individual mutations. Both versions of the analysis software used herein provided the options to designate custom areas of the plot as FAM-positive. Of the three methods for drawing such areas (box, circle and freehand), we found boxing to be easiest to reproduce consistently across separate runs, although it is hoped the future versions of the software will allow precise definition of ellipsoidal regions.

Acceptance criteria for calling mutant positive samples. All ddPCR assays (single- and multiplexes) were optimised in terms of sensitivity, specificity and reproducibility as per the ddPCR assay workflow described in Fig. 3.

In our experience, and even assuming excellent adherence to good pre-PCR practice and sample and reagent separation in the setup of assays, it is high risk to interpret a single positive droplet in a reaction as a "mutation positive" call due to underlying Taq error. We set the requirement that there must be three or more droplets falling within the defined area on the $2 \mathrm{D}$ plot in order to call a sample positive for a particular mutation if all NTC and PTC wells gave the expected result. There is no perfect cut-off to reach a sensitivity (true positive rate) and positive predictive value (PPV or precision) of 1, but it is important for our studies that we do not make false positive calls. Statistical analysis of a reference data set supported thresholding at 3 FAM-positive droplets as optimal. For a test assay using 176 low concentration mutant controls, and $168 \mathrm{WT}$ (mutation negative) controls, this threshold gave a sensitivity of 0.994 and a PPV of 1 (95\% CI [0.973227, 1], data not shown). A threshold of 2 positive droplets led to a PPV of 0.957 ( 8 false positives from 168 true negatives) and a threshold of 4 positive droplets led to a PPV of 1 ( 0 false positives), but with a loss of sensitivity ( 4 false negatives from 172 true positives). In almost all subsequent assays we have found this general observation to remain true and our threshold is never less than 3.

ddPCR cycling conditions. We found that modest changes to default ddPCR cycling conditions had a profound impact on the resulting droplet amplitudes. First reported by Witte et al., and confirmed in this study, we 
a. KRAS multiplex \#1

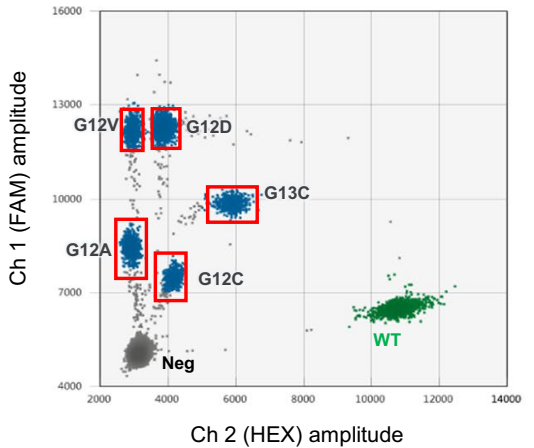

b. KRAS multiplex \#2

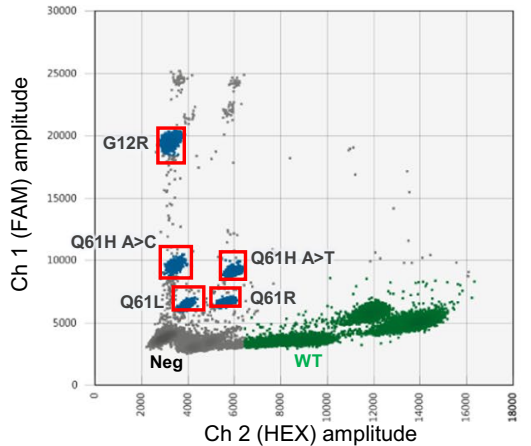

c. KRAS multiplex \#3

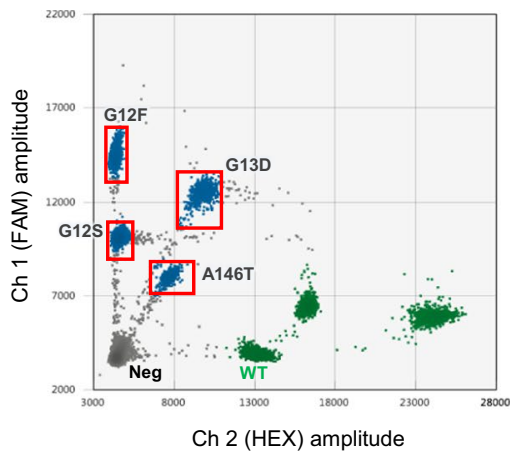

Figure 9. Representative 2D plots of KRAS multiplex ddPCR assays. (a) KRAS multiplex \#1 covering G12A, G12C, G12D, G12V and G13C mutations, (b) KRAS multiplex \#2 covering G12R, Q61H A $>$ C, Q61H A $>$ T, Q61L and Q61R mutations, and (c) KRAS multiplex \#3 covering G12F, G12S, G13D and A146T mutations. Neg - double negative droplets, WT - WT KRAS-positive droplets.

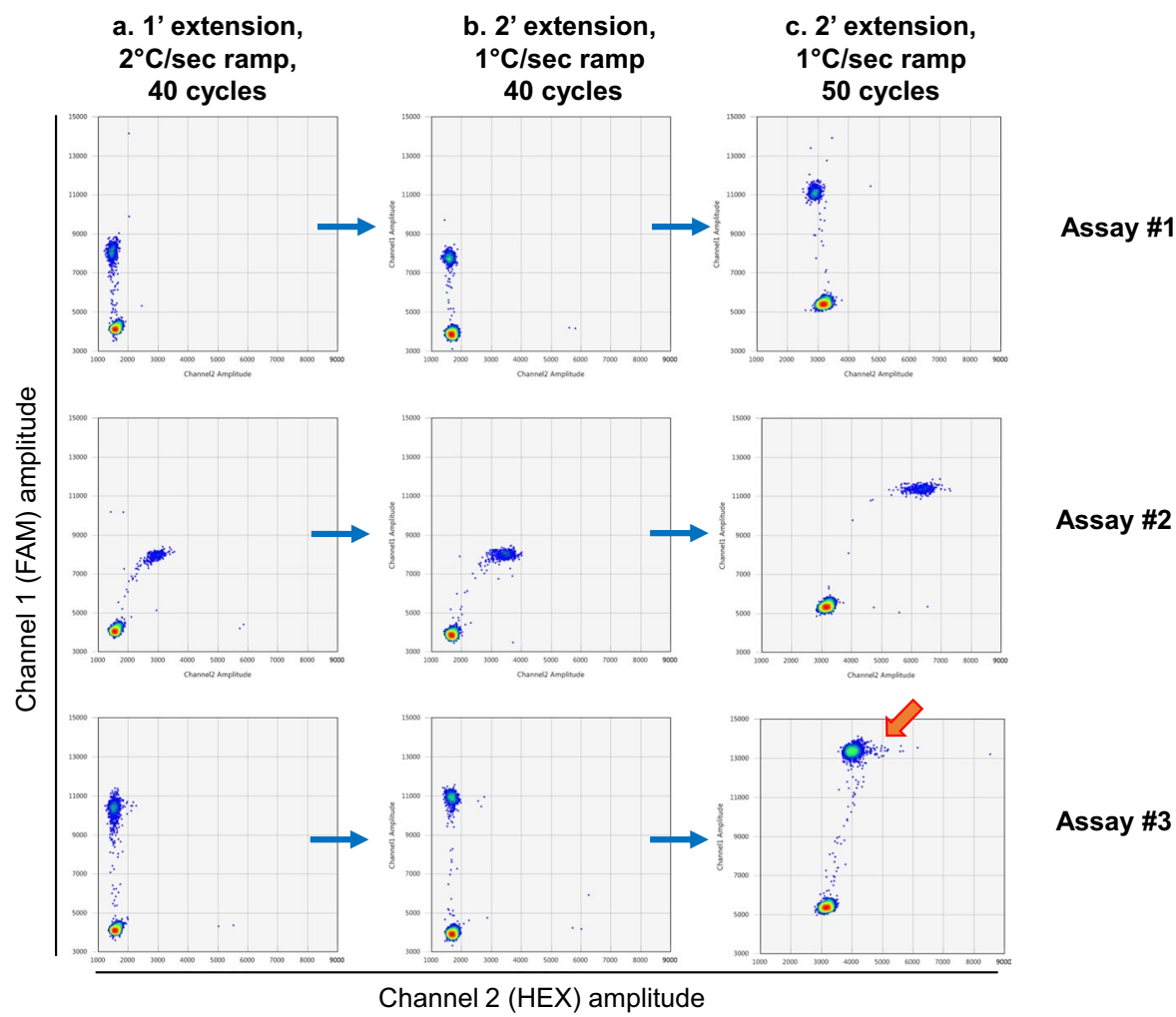

Figure 10. Optimisation of ddPCR cycling conditions. Increasing extension time and reducing ramp rate from (a) standard results in clouds which are (b) more compact with less rain. (c) Rain can be reduced further by increasing the number of PCR cycles from 40 to 50 . Note that altering cycling conditions can change the amplitude of some clouds. Increasing the cycle number up to 50 can lead to the emergence of non-specific double-positive droplets (arrow).

found that the clouds can become more compact and produce substantially less rain upon: (i) reducing the ramp rate to $1^{\circ} \mathrm{C} / \mathrm{sec}$ in every PCR step (Fig. 10a), (ii) increasing the annealing/extension time to 2 minutes (up from 1 minute; Fig. 10b), and (iii) increasing the number of cycles to 50 (Fig. 10c) ${ }^{28}$. However, it should be noted that there can be a trade-off because increasing the cycle number can lead to an increased false-positive rate (additional cycles for Taq error to occur and become detectable).

Additional observations. To achieve optimal accepted droplet number of approximately 20,000 droplets per well, we found that it was important to incubate ddPCR plates on the cycler at $12^{\circ} \mathrm{C}$ for a minimum of 4 hours post cycling and prior to transfer to the droplet reader (Fig. 11). Immediately after PCR, we found there to be 

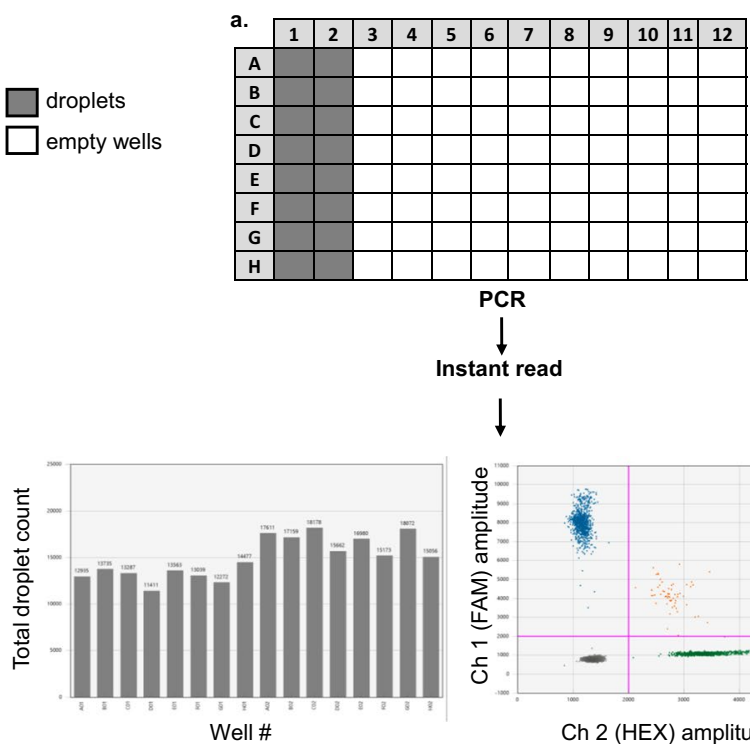

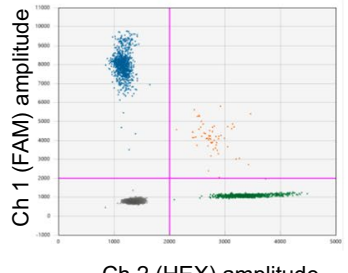

Ch 2 (HEX) amplitude
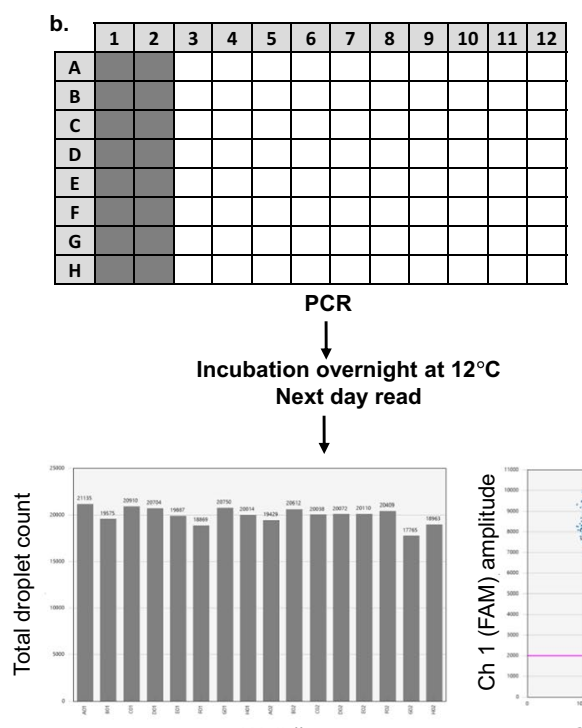

Well \#

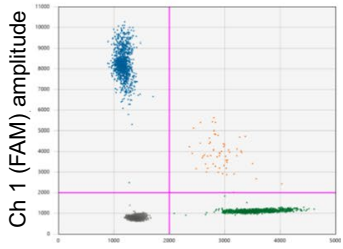

Ch 2 (HEX) amplitude

Figure 11. Incubation of plate at $12{ }^{\circ} \mathrm{C}$ post-PCR prior to droplet reading increases number of accepted droplets. Reading the ddPCR plate (a) immediately after PCR results in fewer total droplets per well compared to (b) incubating the plate overnight at $12{ }^{\circ} \mathrm{C}$ prior to reading with no negative effects on the fluorescent signal.

condensation on the well walls which we believed originated from and lead to shrinkage of the droplets. The QX200 droplet reader, apart from fluorescence intensity, measures the size and shape of each droplet as they pass the detector; droplets are excluded if they do not meet quality metrics (Bio-Rad Bulletin 6407 RevA, page 20). If droplets shrink in size, they can be rejected by the reader resulting in fewer accepted droplets per reaction. Leaving the post-PCR plates at $12^{\circ} \mathrm{C}$ for a minimum of 4 hours allowed the condensation to be reabsorbed into the droplets and lead to significantly higher passing droplet numbers for each well in the plate with no negative effects on the fluorescent signal (Fig. 11). The average droplet number per well was $14913 \pm 2134$ for reading done immediately after PCR (Fig. 11a), and $19953 \pm 864$ for reading done after $>4 \mathrm{~h}$ incubation at $12^{\circ} \mathrm{C}$ (Fig. $11 \mathrm{~b}$ ).

\section{Discussion}

Appropriately designed and executed ddPCR analysis provides for exquisitely sensitive, specific, quantitative, and reproducible detection of mutations in cfDNA, but there are subtleties to its execution that are not necessarily apparent to new users of this technology. Our laboratory has gained several years' experience of assay development and optimisation, and delivery of high quality exploratory data from precious clinical samples in support of translational studies.

Since KRAS mutations are among the most common cancer drivers, ddPCR assays designed to detect them are commercially available, and the development of multiplex ddPCR assays against them has been reported ${ }^{29,30}$. Taly et al., similarly to our approach, used probes targeting the same mutation labelled with two different dyes, which resulted in optimal separation of the clouds on the 2D plot. However, they developed their assays for a different ddPCR platform (by RainDance Technologies) and with non-fluorescent blockers (rather than LNA base-containing oligos) to facilitate discrimination ${ }^{29}$. Pender and colleagues used the approach of mixing commercially available assays for selected KRAS mutations. They achieved good separation of the multiplexed mutations, but their assays often showed non-specific signals. Because the sequence of the probes in these commercial assays is not disclosed, they did not have the option to add to the mixes any probes that would allow to move selected mutant clouds to the right on the $2 \mathrm{D}$ plot. It is also noteworthy that the price of commercially available assays are much higher than the price of in-house designed primers and probes, even when the probe price is high due to the LNA base incorporation. In effect, they were able to combine only up to 3 mutations per multiplex ${ }^{30}$. Because of the above reasons, and because neither of these studies included all 14 KRAS mutation of interest to us, we decided to develop and optimise the multiplexes anew.

In this manuscript, we outline the critical steps we have implemented to ensure our assays deliver the best possible performance whilst minimising sample usage. Figure 2 is a description of key ddPCR data analysis concepts. These characteristics also allow for increased specificity between mutations that may overlap or be on adjacent bases or codons in the same gene locus. All of these features are particularly important when devising multiplexed assays. The resulting assay(s) should be suitable for the detection of the mutation(s) of interest in terms of specificity, sensitivity and reproducibility, with clearly defined criteria for calling a test sample as mutation positive in accordance with the digital MIQE guidelines (Minimum Information for publication of Quantitative dPCR experiments) $)^{31}$.

Diligent and consistent preparation of reagents coupled with good PCR practice can assure a very high reproducibility of the assays. Thorough optimisation of assays, especially multiplexes, as described herein can be very time-consuming. We have found, however, that when it comes to analysis of very limited clinical samples this 
effort pays great dividends in terms of result consistency, quality, and confidence in mutation calls. Introducing changes to the cycling conditions (e.g. increasing extension time, decreasing ramp rate), can produce superior $2 \mathrm{D}$ plots, whilst incubation of the plate at $12{ }^{\circ} \mathrm{C}$ prior to droplet reading can increase the droplet numbers by over $60 \%$. Indeed, in the study by Witte et al. adjusting the cycling conditions greatly improved positive and negative signal separation, but because the authors were focused on fast sample turnaround, they reported droplet numbers as low as 11,000 per well ${ }^{28}$. Since in ddPCR DNA copies are distributed randomly, there is no reason why an assay with e.g. 3 copies would necessarily have them distributed evenly across the populations of droplets that were analysed and not analysed. It is, therefore, only to be expected that some reactions that contained exactly 3 target copies across 20,000 droplets will produce a fraction of results where reading only 15,000 copies will result in 1, 2 or all 3 copies being missed. Not missing extremely rare events is therefore of importance not only in cancer monitoring, but also in detection of other clinically relevant somatic mutations ${ }^{32}$ and other applications such as detection of animal and plant pathogens ${ }^{28,33,34}$.

Some ddPCR assays, when run according to Bio-Rad's standard thermal cycling protocol, can be "rainy" (presence of droplets that are true positives, but appear below the main droplet cluster on 1D or 2D plots). In singleplex assays, this is often not a problem, as the FAM threshold can be placed sufficiently low to encompass most of the rain and thus minimise the number of false negatives. However, some singleplex assays are not only "rainy", but also "misty", i.e. droplets in the HEX-positive (WT/non-mutant) cloud have a tendency for increased FAM amplitude (due to Taq errors), in which case lowering the FAM threshold may lead to an unacceptable level of false positives. Even more critically, in multiplex assays, rain from a cloud representing one mutation can fall in the zone designated for another mutation, resulting in false positives in the latter. Therefore, it is critical to optimise the ddPCR cycling conditions.

In general, when read-out for any sample is lower than 10,000 droplets, we would recommend the sample be repeated (although clearly positive samples may still be called). Although these modifications can increase the turnaround time from sample to result (often making it impossible to carry out the whole procedure in one working day), we consider their benefits to greatly outweigh this small extra time cost.

Reporting the mutation level in copies/ml of original plasma sample has significant advantages over presenting mutation level as an allelic frequency percentage. Allelic frequency is dependent on the total amount of DNA present in the sample, which comprises tumour derived DNA (both mutant and non-mutant at specific loci), normal DNA shed from other sources in the body and also contaminating germline DNA from nucleated blood cells. The level of "contaminating" DNA from each of these sources will vary depending on a variety of factors such as blood sample collection method and tube, storage, handling, processing and stabilising reagents used ${ }^{23,24,35,36}$. Clearly the more non-tumour DNA present, the more an allele frequency estimate for a tumour mutation may be skewed downwards. Likewise, the lower the non-tumour DNA, the higher the apparent allele frequency for a tumour mutation - even when the number of copies of mutant target in a specified volume of plasma are identical. Therefore, a more optimal and unbiased way to describe mutation burden is as mutant copies $/ \mathrm{ml}$ in the original plasma sample because the absolute number of mutant copies should not be influenced by variable levels of contaminating non-tumour DNA (though exceptions to this may occur in cases where plasma is grossly contaminated with very high levels of WT DNA due to poor collection or processing).

Furthermore, the development of the XenT spike-in control allows for monitoring of DNA extraction efficiency within and across batches and for direct DNA extraction efficiency comparison across different extraction platforms. We found no statistically significant difference in the measured extraction efficiency based on the number of copies of the RPP30 gene. Since this assay measures a WT gene, it will produce a positive signal for both cfDNA as well as germline DNA (originating primarily from leukocytes). The extent of this contamination can vary between samples, patient groups, and be dependent on such factors as the anti-coagulant used, differences in collection methods employed etc. Markus et al. compared cfDNA extraction using 7 different commercially available kits ${ }^{24}$. They found great differences in terms of obtained copy number per ml and fraction of low molecular weight DNA. Their study found that among magnetic beads-based kits, MagMAX Nucleic Acid Isolation kits (Thermo Fisher Scientific) produced the highest copy number, but Maxwell RSC kit (Promega) gave the lowest level of high molecular weight DNA contamination. Contrary to their results, we found that a modified protocol for the Mag-Bind cfDNA kit (Omega Bio-Tek) produces the best results (unpublished data). We would therefore recommend users test the quality of the extracted cfDNA and level of its contamination with germline DNA using such platforms as TapeStation or BioAnalyzer. The XenT DNA gBlock used in this study is a fragment of free double-stranded DNA whereas cfDNA in plasma is believed to be bound primarily to nucleosomes, which might result in a different binding efficiency to beads or columns. However, the first steps of cfDNA extraction always include incubation with strong denaturing agent (guanidine thiocyanate) and proteinase $\mathrm{K}$, which serves to release the DNA from nucleosomes.

In summary, we show a few simple ways of improving the validation of ddPCR assays, a way to multiplex them to save precious material, and a method to estimate the extraction efficiency of cfDNA from plasma. We hope our learning will help other labs maximise the performance of their own analyses and further the utility of this platform.

\section{Data Availability}

There are no restrictions on the availability of materials or information.

\section{References}

1. Bettegowda, C. et al. Detection of Circulating Tumor DNA in Early- and Late-Stage Human Malignancies. Sci. Transl. Med. 6, 224ra24-224ra24 (2014).

2. Wan, J. C. M. et al. Liquid biopsies come of age: towards implementation of circulating tumour DNA. Nat. Rev. Cancer 223-238 (2017).

3. Cravero, K. \& Park, B. H. Circulating Tumor DNA-the Potential of Liquid Biopsies. Current Breast Cancer Reports 8, 14-21 (2016). 
4. Crowley, E., Di Nicolantonio, F., Loupakis, F. \& Bardelli, A. Liquid biopsy: monitoring cancer-genetics in the blood. Nat. Rev. Clin. Oncol. 10, 472-484 (2013).

5. Siravegna, G., Marsoni, S., Siena, S. \& Bardelli, A. Integrating liquid biopsies into the management of cancer. Nat. Rev. Clin. Oncol. https://doi.org/10.1038/nrclinonc.2017.14 (2017).

6. Chin, R. et al. Detection of Solid Tumor Molecular Residual Disease (MRD) Using Circulating Tumor DNA (ctDNA). Mol. Diagn. Ther. 23, 311-331 (2019).

7. Pao, W. et al. Acquired resistance of lung adenocarcinomas to gefitinib or erlotinib is associated with a second mutation in the EGFR kinase domain. PLoS Med. 2, e73 (2005).

8. Desai, J. et al. Clonal evolution of resistance to imatinib in patients with metastatic gastrointestinal stromal tumors. Clin. Cancer Res. 13, 5398-5405 (2007).

9. Murtaza, M. et al. Non-invasive analysis of acquired resistance to cancer therapy by sequencing of plasma DNA. Nature 497, 108-112 (2013).

10. Leary, R. J. et al. Development of Personalized Tumor Biomarkers Using Massively Parallel Sequencing. Sci. Transl. Med. 2 , 20ra14-20ra14 (2010).

11. Leary, R. J. et al. Detection of Chromosomal Alterations in the Circulation of Cancer Patients with Whole-Genome Sequencing. Sci. Transl. Med. 4, 162ra154-162ra154 (2012).

12. Chan, K. C. A. et al. Cancer genome scanning in plasma: Detection of tumor-associated copy number aberrations, single-nucleotide variants, and tumoral heterogeneity by massively parallel sequencing. Clin. Chem. 59, 211-224 (2013).

13. Forshew, T. et al. Noninvasive Identification and Monitoring of Cancer Mutations by Targeted Deep Sequencing of Plasma DNA. Sci. Transl. Med. 4, 136ra68-136ra68 (2012).

14. Hindson, B. J. et al. High-throughput droplet digital PCR system for absolute quantitation of DNA copy number. Anal. Chem. 83, 8604-8610 (2011).

15. Sint, D., Raso, L. \& Traugott, M. Advances in multiplex PCR: balancing primer efficiencies and improving detection success. Methods Ecol. Evol. 3, 898-905 (2012).

16. Dawson, S.-J. et al. Analysis of Circulating Tumor DNA to Monitor Metastatic Breast Cancer. N. Engl. J. Med. 368, 1199-1209 (2013).

17. Kang, Q. et al. Comparative analysis of circulating tumor DNA stability In K3EDTA, Streck, and CellSave blood collection tubes. Clin. Biochem. 49, 1354-1360 (2016).

18. You, Y., Moreira, B. G., Behlke, M. A. \& Owczarzy, R. Design of LNA probes that improve mismatch discrimination. Nucleic Acids Res. 34 (2006).

19. Mouliere, F. \& Rosenfeld, N. Circulating tumor-derived DNA is shorter than somatic DNA in plasma. Proc. Natl. Acad. Sci. 112, 3178-3179 (2015).

20. Underhill, H. R. et al. Fragment Length of Circulating Tumor DNA. PLoS Genet. 12 (2016).

21. Pérez-Barrios, C. et al. Comparison of methods for circulating cell-free DNA isolation using blood from cancer patients: impact on biomarker testing. Transl. Lung Cancer Res. 5, 665-672 (2016).

22. Devonshire, A. S. et al. Towards standardisation of cell-free DNA measurement in plasma: Controls for extraction efficiency, fragment size bias and quantification. Anal. Bioanal. Chem. 406, 6499-6512 (2014).

23. Risberg, B. et al. Effects of Collection and Processing Procedures on Plasma Circulating Cell-Free DNA from Cancer Patients. J. Mol. Diagnostics, https://doi.org/10.1016/j.jmoldx.2018.07.005 (2018).

24. Markus, H. et al. Evaluation of pre-analytical factors affecting plasma DNA analysis. Sci. Rep. 8 (2018).

25. Diefenbach, R. J., Lee, J. H., Kefford, R. F. \& Rizos, H. Evaluation of commercial kits for purification of circulating free DNA. Cancer Genet. https://doi.org/10.1016/j.cancergen.2018.08.005 (2018).

26. Suzawa, K. et al. Optimal method for quantitative detection of plasma EGFR T790M mutation using droplet digital PCR system. Oncol. Rep. 37, 3100-3106 (2017).

27. Hortobagyi, G. N. et al. Correlative analysis of genetic alterations and everolimus benefit in hormone receptor-positive, human epidermal growth factor receptor 2-negative advanced breast cancer: Results from BOLERO-2. J. Clin. Oncol. 34, 419-426 (2016).

28. Witte, A. K. et al. A systematic investigation of parameters influencing droplet rain in the Listeria monocytogenes prfA assay -reduction of ambiguous results in ddPCR. PLoS One 11 (2016).

29. Taly, V. et al. Multiplex picodroplet digital PCR to detect KRAS mutations in circulating DNA from the plasma of colorectal cancer patients. Clin. Chem. 59, 1722-1731 (2013).

30. Pender, A. et al. Efficient genotyping of KRAS mutant non- small cell lung cancer using a multiplexed droplet digital PCR approach. PLoS One 10 (2015)

31. Huggett, J. F. et al. The digital MIQE guidelines: Minimum information for publication of quantitative digital PCR experiments. Clin. Chem. 59, 892-902 (2013).

32. Uchiyama, Y. et al. Ultra-sensitive droplet digital PCR for detecting a low-prevalence somatic GNAQ mutation in Sturge-Weber syndrome. Sci. Rep. 6 (2016).

33. Tang, H., Cai, Q., Li, H. \& Hu, P. Comparison of droplet digital PCR to real-time PCR for quantification of hepatitis B virus DNA. Biosci. Biotechnol. Biochem. 80, 2159-2164 (2016).

34. Selvaraj, V. et al. Development of a duplex droplet digital PCR assay for absolute quantitative detection of 'Candidatus Liberibacter asiaticus'. PLoS One 13, e0197184 (2018).

35. Norton, S. E., Lechner, J. M., Williams, T. \& Fernando, M. R. A stabilizing reagent prevents cell-free DNA contamination by cellular DNA in plasma during blood sample storage and shipping as determined by digital PCR. Clin. Biochem. 46, 1561-1565 (2013).

36. Norton, S. E., Luna, K. K., Lechner, J. M., Qin, J. \& Fernando, M. R. A new blood collection device minimizes cellular DNA release during sample storage and shipping when compared to a standard device. J. Clin. Lab. Anal. 27, 305-311 (2013).

\section{Acknowledgements}

We would like to gratefully thank Dr. Nitzan Rosenfeld, Dr. Davina Gale, Dr. Dana Tsui and Dr. Tim Forshew (Cancer Research UK Cambridge Institute) for generously providing the initial Xenopus tropicalis sequence and for describing the principle of using a non-human genomic target as an internal control for DNA extraction efficiency, an adaptation of which we present here. We would also like to thank Dr. Miika Ahdesmaki for additional statistical analysis and support.

\section{Author Contributions}

V.R., A.R. and T.C. devised the experiments. V.R. and A.R. performed the experiments. V.R., A.R. and E.M. analysed data wrote the manuscript and prepared figures. All authors reviewed the manuscript.

Additional Information

Supplementary information accompanies this paper at https://doi.org/10.1038/s41598-019-49043-X. 
Competing Interests: The authors declare no competing interests.

Publisher's note: Springer Nature remains neutral with regard to jurisdictional claims in published maps and institutional affiliations.

(c) (i) Open Access This article is licensed under a Creative Commons Attribution 4.0 International cc) License, which permits use, sharing, adaptation, distribution and reproduction in any medium or format, as long as you give appropriate credit to the original author(s) and the source, provide a link to the Creative Commons license, and indicate if changes were made. The images or other third party material in this article are included in the article's Creative Commons license, unless indicated otherwise in a credit line to the material. If material is not included in the article's Creative Commons license and your intended use is not permitted by statutory regulation or exceeds the permitted use, you will need to obtain permission directly from the copyright holder. To view a copy of this license, visit http://creativecommons.org/licenses/by/4.0/.

(C) The Author(s) 2019 